\title{
PROCESSO DE ESCOLHA DA MELHOR LOCALIZAÇÃO DE UNIDADES OPERACIONAIS MÓV̉EIS: UMA APLICAÇÃO DA TÉCNICA PROCESSO DE ANÁLISE HIERÁRQUICA - AHP
}

\author{
APPLYING AHP TO THE PROCESS OF \\ CHOOSING THE BEST COMBINATION OF \\ LOCATIONS OF MOBILE OPERATIONAL UNITS
}

Recebido 30-09-2012

Aceito 21-02-2013

Denise Lindstrom Bandeira ${ }^{1}$

João Luiz Becker ${ }^{2}$

Thiago Ramires Eichenberg ${ }^{3}$

\section{RESUMO}

Este trabalho apresenta a aplicação da técnica Processo de Análise Hierárquica (AHP) para auxiliar o processo decisório de uma pequena empresa, que desenvolveu e usa uma nova tecnologia móvel para o tratamento térmico de pallets de madeira. A decisão refere-se à escolha da melhor combinação de locais para instalar as três bases operacionais da empresa no estado do Rio Grande do Sul. Como o tratamento térmico deve ser sempre realizado nas instalações dos clientes, a localização das bases operacionais é uma decisãochave para a sobrevivência da empresa, assim como para seu crescimento, pois se relaciona diretamente com várias outras importantes variáveis, como custos operacionais, oferta e treinamento de mão de obra especializada, tempo de resposta às solicitações dos clientes, etc. O portfólio de clientes da empresa vem crescendo consideravelmente devido à inovadora tecnologia usada no processo de esterilização dos pallets. Há mais de 50 clientes espalhados em uma área superior a $250.000 \mathrm{~km}^{2}$. Para a diretoria à frente do empreendimento, analisar o problema exclusivamente sob a tradicional ótica de custos pareceu insuficiente. A utilização da técnica AHP proporcionou importantes informações, permitindo incorporar outros fatores na análise, além da visão estritamente financeira. Os fatores envolvidos, sua estrutura hierárquica e seus pesos relativos foram estabelecidos por consenso entre os gerentes e executivos da empresa.

Palavras-chave: Tratamento térmico de pallets. Processo de decisão em grupo. Problema da localização. Controle de pestes.

\footnotetext{
${ }^{1}$ Possui graduação em Geologia e Ciência da Computação pela Universidade Federal do Rio Grande do Sul- UFRGS, mestrado e doutorado em Administração pela Universidade Federal do Rio Grande do Sul- UFRGS. Atualmente é professora adjunta de Administração da Universidade Federal do Rio Grande do Sul- UFRGS. Porto Alegre, Rio Grande do Sul, Brasil. E-mail: dlbandeira@ea.ufrgs.br.

2 Possui graduação em Ciências Econômicas e Matemática pela Universidade Federal do Rio Grande do Sul- UFRGS, mestrado em Matemática Aplicada pelo Instituto Nacional de Matemática Pura e Aplica - IMPA. Doutorado em Management Science pela University of California. Atualmente é professor titular de Administração da Universidade Federal do Rio Grande do Sul - UFRGS. Porto Alegre, Rio Grande do Sul, Brasil. E-mail: jlbecker@ea.ufrgs.br.

${ }^{3}$ Possui graduação em Administração pela Universidade Federal do Rio Grande do Sul- UFRGS. Porto Alegre, Rio Grande do Sul, Brasil. E-mail: thiago.eichenberg@fitolog.com.br.
} 


\section{ABSTRACT}

This paper presents the application of the Analytic Hierarchic Process (AHP) technique in helping a decision-making process of a young and small sized company that developed and currently make use of a new mobile technology for the heat treatment of wooden packaging and pallets. The decision process comprised choosing the best combination of locations to place the company's three operational bases. As the heat treatment service must always be provided at the customers' premises, this decision is crucial to the company survival, as well as for its growth, as it influences a large number of important variables, such as operational costs, supply and training of skilled manpower, response time to costumer's requests, and others. The company's portfolio of clients is rising firmly because of the innovative technology used in the process of sterilization. It is currently formed by more than 50 customers spread within a 100,000 square miles area. For the CEO, thinking exclusively in terms of costs appeared to be insufficient. That's why the AHP was able to provide relevant inputs in this case, by incorporating other factors beyond the purely financial view in its analysis. The factors involved, their hierarchical structure and relative weights, were generated in consensus by the company's managers and executives' opinion.

Keywords: Heat treatment of pallets. Group decision making. Location problem. Pest control.

\section{INTRODUÇÃO}

O problema de localização representa uma das mais importantes questões estratégicas enfrentadas pelas organizações, tanto públicas como privadas (FARAHANI et al., 2012). Decidir onde localizar uma fábrica, um ponto de venda, ou a base operacional de unidades móveis é sempre uma decisão-chave. Tanto a seleção dos critérios de julgamento como a própria avaliação das possíveis alternativas são elementos críticos no processo de escolha da melhor localização. Este trabalho apresenta uma aplicação da técnica Processo de Análise Hierárquica (AHP - do inglês Analytic Hierarchic Process) (SAATY, 1980) para auxiliar o processo decisório de seleção das meIhores cidades para instalar bases para as unidades operacionais móveis da Fitolog (2012a), uma pequena empresa em acelerado processo de crescimento. A intenção é demonstrar a possibilidade de analisar critérios fora da visão meramente financeira, levando em consideração outros fatores, gerenciais e organizacionais, que possam melhorar a decisão. A utilização da técnica AHP tem permitido aos administradores a criação de cenários para situações inexistentes, que podem auxiliar na tomada de decisão. Essa aplicação em situação empresarial típica tem por objetivo selecionar critérios que contribuam estrategicamente para avaliar as localizações alternativas existentes, possibilitando, assim, uma melhor escolha.

O estudo limita-se ao caso da empresa analisada, principalmente pela disponibilidade de dados para efetuar as análises e entrevistar os gerentes responsáveis pela decisão. Mas o método desenvolvido poderá ser estendido para outros problemas de localização. $O$ artigo se organiza da seguinte forma: a seção 2 apresenta a contextualização do estudo e a situação problemática; a seção 3 descreve a técnica de solução utilizada, apresentando o método na seção 4; a seção 5 traz o desenvolvimento do modelo e na seção 6 são analisados os resultados obtidos.

\section{CONTEXTUALIZAÇÃO DO ESTUDO}

A empresa na qual o estudo foi realizado é uma companhia com pouco mais de três anos de operação que tem crescido rapidamente (FITOLOG, 2012b). Operando no mercado de controle de pestes, mais de $90 \%$ de suas vendas provêm do tratamento fitossanitário de pallets de madeira utilizados em exportações a partir do Estado do Rio Grande do Sul, atendendo à legislação do comércio internacional, como a norma ISPM 15. A empresa se diferencia de seus com- 
petidores por utilizar uma tecnologia única de tratamento térmico, integralmente desenvolvido pela empresa, chamada de Processo de Câmara Portátil Fitolog - PCP Fitolog (Figura 1) - com processo de patenteamento encaminhado ao Instituto Nacional da Propriedade Industrial (INPI).

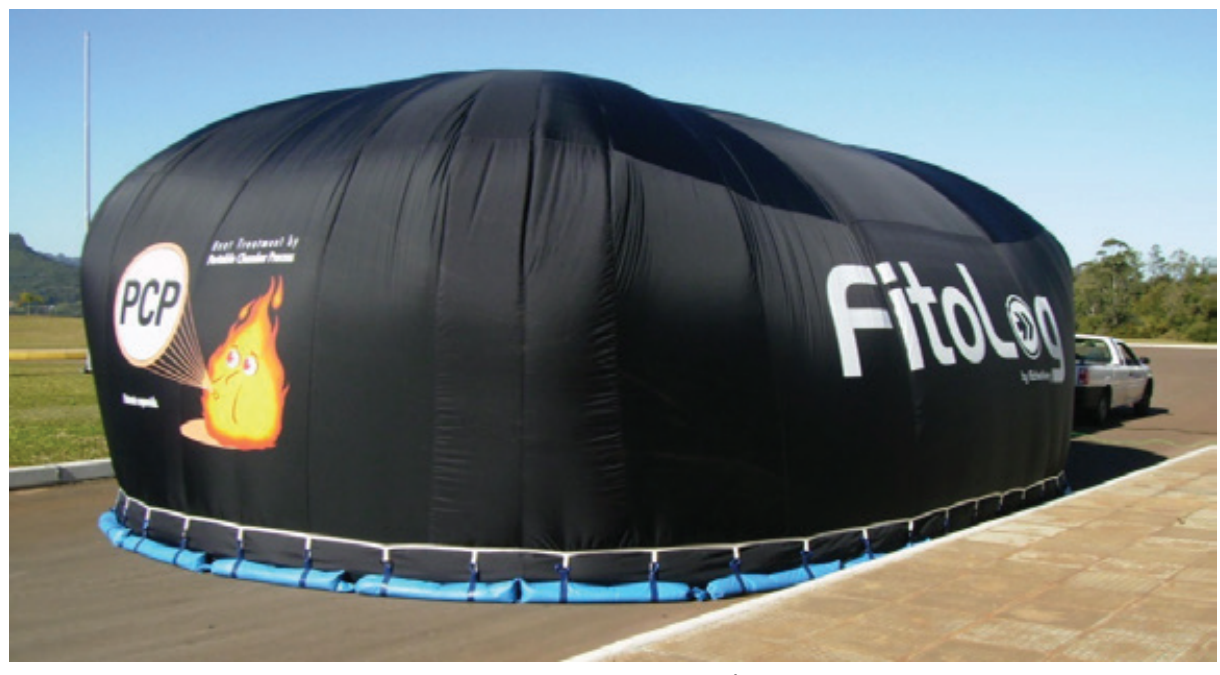

Figura 1 - Processo de tratamento térmico da Fitolog Fonte: Fitolog (2012a)

Anteriormente ao desenvolvimento do PCP Fitolog, os exportadores gaúchos tinham apenas duas maneiras de tratar suas embalagens e pallets de madeira para exportação:

- Fumigação com Metil Bromido (MBF - do inglês Methyl Bromide Fumigation) - Procedimento químico realizado pela aplicação do gás pesticida Metil Bromido (MB - do inglês Methyl Bromide) diretamente na embalagem ou pallet de madeira. Após receber a aplicação do $\mathrm{MB}$, a embalagem deve ser colocada em quarentena por 24 horas. A grande desvantagem deste procedimento é o dano ambiental causado, pois o MB reage com o ozônio na atmosfera. Por esta razão, este tipo de tratamento está proibido de ser utilizado a partir do ano de 2015 (o uso de MB na agricultura já está abolido há algum tempo). $O$ tratamento MBF tem, entretanto, uma grande vantagem logística, pois pode ser realizado em qualquer lugar, pois o equipamento necessário é bastante compacto, podendo ser montado em veículos utilitários leves.

- Tratamento Térmico Padrão (SHT - do inglês Standard Heat Treatment) - Este tipo de tratamento, além de ser mais rápido (com tempo médio variando de duas a três horas) não agride o meio ambiente, pois não utiliza pesticidas. Apenas calor, é usado para eliminar as pestes. De acordo com a norma ISPM 15, para ser aceito como válido, um tratamento térmico deve elevar a temperatura da embalagem ou pallet de madeira até $56^{\circ} \mathrm{C}$, permanecendo nesta temperatura por pelo menos 30 minutos. A principal desvantagem do SHT é a sua relativa imobilidade, pois os fornos de tratamento são em geral estruturas grandes e pesadas. Como consequência, o cliente acaba arcando com os custos de transporte de suas embalagens ou pallets de madeira para o local onde o tratamento será realizado.

A tecnologia desenvolvida pela Fitolog, o PCP Fitolog, objetivou amealhar as vantagens de ambos os tratamentos. Além de ser um tratamento não agressivo ao meio ambiente, o PCP Fitolog pode ser usado em qualquer lugar, substituindo os fornos estáticos por câmaras portáteis, feitas com um tecido térmico especial, pesando cerca de $30 \mathrm{~kg}$ e conectadas a um módulo aquecedor compacto que pode ser transportado ao local de utilização em camionetes leves, oferecendo, portanto, ampla mobilidade ao processo.

Ao combinar alta tecnologia com custos baixos, a Fitolog pode oferecer aos seus clien- 
tes um serviço bastante conveniente. Por esta razão, a empresa conquistou rapidamente uma boa fatia de mercado, tornando-se já em 2011 a empresa líder no segmento de grandes exportadores do sul do país, com mais de $35 \%$ de "market-share". Como resultado, suas receitas se multiplicaram por quatro em 2011 (em relação a 2010).

Devido a este rápido crescimento, o portfólio da empresa já conta com mais de 50 grandes clientes espalhados em uma área de mais de $250.000 \mathrm{~km}^{2}$. Apesar da grande extensão territorial, as três unidades operacionais móveis da empresa continuavam baseadas junto à sua sede, em Porto Alegre, estado do Rio Grande do Sul. Mas esta condição logo começou a ser questionada pelos executivos da empresa, imaginando se não seria mais interessante distribuir as unidades operacionais móveis para outras cidades. Cinco localidades, incluindo Porto Alegre, foram pré-selecionadas como aptas a sediarem unidades operacionais móveis, em parceria com empreendedores locais.

Na literatura especializada, problemas de localização têm sido tradicionalmente abordados sob a ótica estritamente econômica, buscando escolher a alternativa que maximize (ou minimize) alguma função objetivo. Hale e Moberg (2003) definem problemas de localização de instalações como aqueles que investigam onde localizar fisicamente um conjunto de instalações (recursos), de modo a minimizar o custo de satisfação de algum conjunto de demandas (clientes) sujeita a algum conjunto de restrições. Mais genericamente, como apontam ReVelle e Eiselt (2005), o termo análise de localização (do inglês location analysis) diz respeito a modelagem, formulação e solução de uma classe de problemas (em pesquisa operacional) que podem ser abstratamente descritos como "localizar unidades em algum espaço". $\mathrm{O}$ assunto tem chamado a atenção de vários pesquisadores há muito tempo, os autores mencionam a existência de milhares de pesquisas publicadas e aplicadas em várias situações específicas. Diversos artigos apresentam compilações de trabalhos publicados no tema, sob distintas óticas, como Mesa e Boffey (1996), Bryan e O'Kelly (1999), Hale e Moberg (2003), ReVelle e Eiselt (2005), Snyder (2006), Şahin e Süral (2007), ReVelle, Eiselt e Daskin (2008), Melo, Nickel e Saldanha-daGama, (2009), Farahani et al. (2012). Todos eles reforçam a ideia de que o problema tem sido tratado essencialmente sob a ótica de otimização, as técnicas mais utilizadas sendo programação linear inteira ou mista. ReVelle e Eiselt (2005) admitem, entretanto, que o problema de localização, encontrando-se na ponta superior da clássica segmentação de decisões como estratégicas-táticas-operacionais, são tipicamente nebulosos e mal caracterizados, e consequentemente muito mais difíceis de ser modelados. Admitem os autores que a abordagem de análise de decisões parece promissora, mencionando o trabalho de Rey et al. (1995, apud REVELLE; EISELT, 2005), que apresentam uma abordagem iterativa incorporando técnicas multicriteriais para definir a localização de um depósito de dejetos.

Yang e Lee (1997) salientam que a decisão sobre a localização de instalações é tipicamente um problema de decisão multicriterial, na qual as preferências dos decisores entre os critérios de performance representam um elemento-chave na decisão final. Mencionam ainda que as abordagens mais utilizadas são: a teoria da utilidade com múltiplos atributos e a técnica AHP. De fato, a técnica AHP tem sido utilizada para resolver o problema de localização por vários autores (YURIMOTO; MASUI, 1995; KORPELA; TUOMINEN, 1996; YANG; LEE, 1997; BADRI, 1999; KUO; CHI; KAO, 1999; ATTHIRAWONG; MACCARTHY, 2002; SAWICKA; WĘGLIŃSKI; WITORT, 2010; PORTUGAL; MORGADO; LIMA JR, 2011).

Neste artigo, será utilizada a técnica AHP como fio condutor de um processo de decisão em grupo. Cada cidade tem suas vantagens ou desvantagens em relação às demais, com respeito a cada critério (ou subcritério), de modo que a escolha entre as possibilidades não é simples, não havendo uma alternativa dominante (isto é uma cidade melhor do que as demais em todos os critérios). A utilização da técnica AHP tornou possível a comparação cruzada entre aspectos tanto qualitativos como quantitativos. 


\section{O PROCESSO DE ANÁLISE HIERÁRQUICA}

O Processo de Análise Hierárquica (AHP - do inglês Analytic Hierarchy Process) é uma técnica de análise de decisão e solução de problemas complexos, envolvendo múltiplos critérios, desenvolvida por Thomas L. Saaty em meados da década de 1970 (GOLDEN; WASIL; HARKER, 1989). Bandeira, Becker e Rocha (2010) salientam que o AHP baseia-se na relativa facilidade que a mente humana possui de estabelecer comparações entre dois objetos dentro de uma mesma classe (comparações pareadas). Buscam-se não apenas comparações ordinais (qual dos dois objetos é mais importante? qual é o preferido?), mas, sobretudo, cardinais (quanto o objeto $\mathrm{A}$ é mais importante do que o objeto B? quão mais preferível?). Saaty (1980) sugere a utilização de uma escala de razão, representando a intensidade em importância ou preferência relativa de nove pontos, com o valor 1 significando "igualmente importante ou preferido" e 9 significando "extremamente mais importante ou preferido". Valores de 2 a 8 representam relações de importância ou preferência em intensidades intermediárias.

Dado um grupo de objetos, uma classificação de todos os seus objetos pode então ser obtida a partir da combinação das comparações pareadas entre todos os objetos do grupo (tomados dois a dois), com eventuais inconsistências encontradas (devido à multiplicidade de comparações pareadas entre todos os objetos do grupo, realizadas separadamente) sendo interpretadas como erros do processo cognitivo. Erros são geralmente eliminados tomando alguma conveniente média dos resultados das comparações pareadas. Saaty (1980) sugere colocar os resultados das comparações pareadas dos objetos em uma matriz quadrada, digamos aij] , cujas linhas e colunas representam os objetos sob avaliação. A matriz será positiva e recíproca, isto é, com todos seus elementos positivos, com aii=1 e aij=1/aji e . 0 autovetor normalizado associado ao maior autovalor da matriz A oferece então os escores de importância ou preferência de cada objeto. Evidentemente, inconsistências muito acentuadas invalidarão o processo, sugerindo-se neste caso uma reelaboração das comparações pareadas, em um processo de retroalimentação informacional. Saaty sugere um índice para medir a inconsistência da matriz de comparações pareadas, o índice de inconsistência ( $\mathrm{Cl}$ - do inglês consistency index), definido por ?]max-nn-1, onde ?max é o maior autovalor da matriz de comparações pareadas e $n$ é o número de objetos no grupo (isto é, a ordem da matriz de comparações pareadas). Uma comparação do $\mathrm{Cl}$ de uma dada matriz de comparações pareadas com índices de consistência médios de matrizes de mesma ordem geradas aleatoriamente produz uma medida denominada razão de consistência ( $C R$ - do inglês consistency ratio), utilizada para aferir a qualidade geral do processo de julgamento. Valores de CR menores ou iguais a 0,1 são considerados aceitáveis. Valores superiores a 0,1 exigem a atenção dos avaliadores no sentido de reduzir suas inconsistências de julgamento, revisando tais valores devidamente (SAATY, 1980).

A aplicação da técnica é dividida em três fases: Estruturação, Avaliação e Síntese. Estruturação refere-se ao desenvolvimento de uma estrutura hierárquica, que modela as relações entre o objetivo da decisão (primeiro nível), critérios que traduzem o objetivo (segundo nível), subcritérios que qualificam cada critério (terceiro nível), se necessários, subsubcritérios que qualificam mais refinadamente cada subcritério (níveis intermediários), se necessários, etc., e alternativas de decisão (último nível), como esquematizado na Figura 2. 


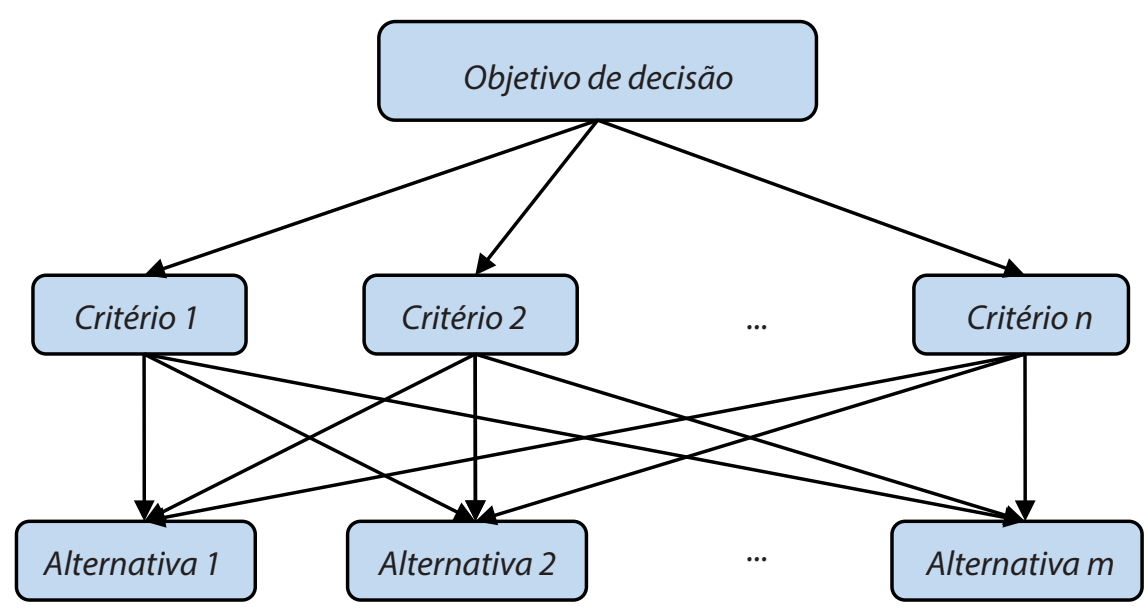

ALTERNATIVAS

Figura 2 - Modelo hierárquico utilizado pela técnica AHP Fonte: Adaptado de Bandeira, Becker e Rocha (2010).

Avaliação refere-se aos julgamentos de valor (comparações pareadas) que deverão ser estabelecidos entre os objetos representados na estrutura hierárquica dentro de cada grupo de referência. Isto é, entre as diferentes alternativas com respeito a cada subcritério ou critério, entre os diferentes subcritérios com respeito ao critério do qual são qualificadores, e entre os diferentes critérios com respeito ao objetivo. Tais comparações pareadas são representadas por matrizes quadradas positivas e recíprocas, como as apresentadas na Seção 5.2.

Síntese refere-se à obtenção de escores cardinais de importância ou preferência de cada alternativa de decisão. Inicia-se determinando os escores cardinais de importância ou preferência de cada objeto representado na estrutura hierárquica, dentro de seu grupo de referência, a partir das comparações pareadas entre todos os objetos do grupo (tomados dois a dois) representados em matrizes quadradas positivas e recíprocas. Como já salientado, para cada matriz de comparações pareadas, o autovetor normalizado associado ao seu maior autovalor oferece os escores cardinais de importância ou preferência de cada objeto. Avalia-se também a inconsistência de cada grupo de comparações, retomando o processo de avaliação, se necessário. A seguir, com os escores cardinais de importância ou preferência de cada objeto representado na estrutura hierárquica, procede-se à determinação dos escores finais de cada alternativa de decisão, utilizando os escores cardinais de importância ou preferência dos objetos nos níveis mais elevados da estrutura hierárquica como coeficientes de composições lineares dos escores cardinais de importância ou preferência dos objetos imediatamente abaixo na hierarquia, em um processo recorrente, definindo assim os escores finais de cada alternativa.

Na medida em que o modelo hierárquico precede e condiciona as demais fases do processo, de avaliação e de síntese, é fundamental que ele esteja bem estabelecido pelo decisor ou decisores, antes que a segunda fase seja iniciada. Especificamente, os decisores devem se assegurar de que a estrutura hierárquica seja completa e não redundante. Uma hierarquia completa contém todas as alternativas e todos os critérios importantes para a decisão, os critérios talvez qualificados por subcritérios, se necessário. Uma hierarquia não redundante é aquela na qual não há sobreposição semântica entre critérios e subcritérios, assim como entre alternativas. Isto é, cada critério ou subcritério contempla isolada e especificamente um determinado significado julgado importante ao processo decisório, e não há alternativas duplicadas. Adicionalmente, a técnica exige que os critérios (e seus respectivos subcritérios, se houver) sejam independentes entre si, pois os escores de importância ou preferência dos objetos nos níveis mais elevados da 
estrutura hierárquica serão utilizados como coeficientes de composições lineares dos escores de importância ou preferência dos objetos imediatamente abaixo na hierarquia, em um processo recorrente, definindo assim os escores finais de cada alternativa.

A simplicidade do processo permite avaliar fatores qualitativos e quantitativos, tangíveis ou intangíveis, aliada à sua fundamentação matemática, de fácil programação em computadores, explica a sua popularidade. O AHP é hoje uma das técnicas mais conhecidas e utilizadas tanto no Brasil como no resto do mundo (GOMES; ANDRADE, 2012). Vaidya e Kumar (2006) e Ho (2008) apresentaram recentes compilações de aplicações internacionais desta técnica.

Uma revisão dos principais periódicos brasileiros (a partir da base Scielo ${ }^{4}$ e do portal de periódicos da CAPES $^{5}$ ) revela que a técnica AHP tem sido usada por pesquisadores brasileiros para avaliar tanto o desempenho de indivíduos (GOMES; ANDRADE, 2012) como o desempenho de organizações (IAÑEZ; CUNHA, 2006; LUZ; SELLITTO; GOMES, 2006; ROSA; SELLITTO; MENDES, 2006; SELLITTO; MENDES, 2006; SELLITTO; WALTER, 2006; RAFAELI; MÜLLER, 2007; VENTURA; REIS; TAKAYANAGUI, 2010), assim como o desempenho de produtos (FOGLIATTO; ALBIN, 2001); para avaliar a gravidade de irregularidades de falhas em processos industriais (AGUIAR; SALOMON, 2007), o risco de setores econômicos (GARTNER; MOREIRA; GALVES, 2009), a potencialidade hídrica de bacias hidrográficas (RIBEIRO; ROCHA; GARCIA, 2011) e a vulnerabilidade ambiental de áreas urbanas (CARVALHO; RIEDEL, 2004; CAVALCANTE et al., 2010) e florestais (VALENTE; VETTORAZZI, 2008); para estabelecer prioridades em embarques marítimos (BANDEIRA; BECKER; ROCHA, 2010), em competição em cadeias de suprimento (SILVA et al., 2012), de fatores críticos de sucesso em projetos (DE CARLI; DELAMARO; SALOMON, 2010), e de recursos e competências do setor nucleoenergético brasileiro (MACEDO-SOARES; FIGUEIRA, 2007); para selecionar projetos (TORTORELLA; FOGLIATTO, 2008; PADOVANI; CARVALHO; MUSCAT, 2010; BARIN et al., 2011; BERNARDON et al., 2011), tecnologias (LAURINDO et al., 2002; MARCHEZETTI; KAVISKI; BRAGA, 2011; RIBEIRO; PASSOS; TEIXEIRA, 2012), programas públicos (ABREU et al., 2000; LIMA; RAMOS; FERNANDES JR., 2008), áreas favoráveis ao cultivo de café (BARROS; MOREIRA; RUDORFF, 2007) e à mineração (CUNHA; OLIVEIRA; SILVA, 2001), variedades de cana-de-açúcar para plantio (COSTA; MOLL, 1999) e objetivos comuns em redes de cooperação (TÁLAMO; CARVALHO, 2004); para determinar o custo de capital próprio de empresas de capital fechado (MATOS; MOURA, 2003) e para localização de terminais de carga (PORTUGAL; MORGADO; LIMA JR, 2011). Cruz Jr. e Carvalho (2003) a utilizam para desenvolver um instrumento de coleta de informações sobre a satisfação do consumidor. Campos, Ramos e Correia (2008) a usam para desenvolver seu índice de sustentabilidade de mobilidade urbana. Corso e Löbler (2011) a usam como base de seu desenho experimental para melhor entender a influência da pressão do tempo e da ausência de informação no processo decisório individual. Reis e Löbler (2012) a usam como base de comparação entre decisões tomadas com e sem apoio de um sistema de apoio à decisão. $O$ banco de teses e dissertações da CAPES $^{6}$ informa que mais de uma centena de teses e dissertações foram desenvolvidas utilizando AHP. Observa-se que a técnica tem sido utilizada isoladamente ou em conjunto com outras técnicas de análise.

\section{MÉTODO}

Este estudo usou como base a metodologia de resolução de problemas, desenvolvida no âmbito da pesquisa operacional (ACKOFF; SASIENI, 1968; WAGNER, 1986), com a proposta de

\footnotetext{
${ }^{4}$ www.scielo.org

${ }^{5}$ www.capes.gov.br/periodicos

${ }^{6} \mathrm{http}: / /$ capes.gov.br/servicos/banco-de-teses
} 
empregar um modelo matemático para auxiliar o processo decisório na seleção das melhores cidades para a instalação de bases para as unidades operacionais móveis de uma pequena empresa prestadora de serviços de controle de pestes. Foram utilizadas as etapas clássicas de um estudo de pesquisa operacional: formulação do problema (onde localizar as unidades operacionais móveis fora da visão estrita de análise financeira); construção do modelo (ver seção 5.1); solução do modelo (método AHP, utilizando planilha eletrônica, ver seções 5.2 e 5.3); teste do modelo e avaliação da solução (validação de face com executivos da empresa, ver seção 5.4); e avaliação final (ver seção 6).

\section{DESENVOLVIMENTO DO MODELO}

Esta seção descreve a construção do modelo de avaliação multicritério para localizar as unidades operacionais móveis da Fitolog, através da técnica AHP.

\subsection{Construção da estrutura hierárquica}

A partir de uma revisão da literatura (Fleury; Wanke; Figueiredo, 2000; Moreira, 2008), sete critérios foram identificados como importantes para a seleção da melhor localização das unidades operacionais móveis da empresa. Os critérios foram agrupados por relevância, segundo um grupo de executivos da empresa e rotulados como: (1) mão de obra, (2) aspectos administrativos (parcerias e controle administrativo), (3) custo de insumos (GLP e óleo diesel (PREÇO DOS COMBUSTÍVEIS, 2010)) e (4) geografia (proximidade da assistência técnica e proximidade de mercados consumidores). Os critérios usados estão representados na Figura 3.

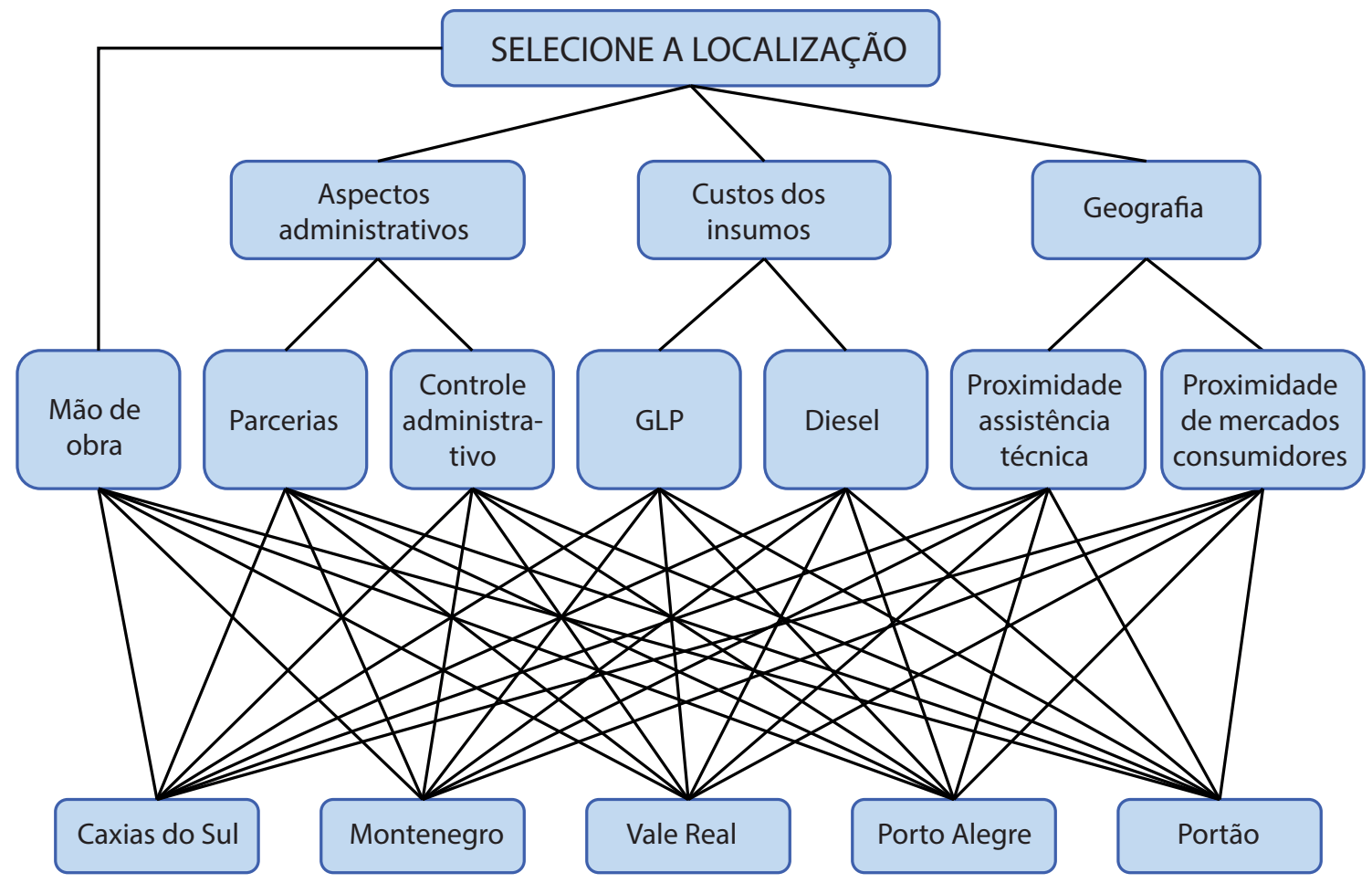

Figura 3 - Estrutura hierárquica para o problema de localização das unidades operacionais móveis da Fitolog Fonte: elaborada pelos autores. 
O objetivo no primeiro nível é definido pela questão: qual cidade deve ser escolhida para abrigar uma unidade operacional móvel da Fitolog? Os critérios e subcritérios foram validados em reuniões sucessivas com um grupo de três executivos da Fitolog: o executivo-chefe da empresa, o gerente administrativo-financeiro e o gerente comercial. Ao final do processo os quatro critérios, mão de obra, aspectos administrativos, custo dos insumos, e geografia, emergiram por consenso entre todas as pessoas envolvidas.

Mão de Obra refere-se ao custo de contratação de funcionários operacionais com mínimo conhecimento de eletricidade. Alguns polos industriais elevam o piso salarial, aumentando a competição por mão de obra qualificada. A importância deste fator não foi alta, considerando a qualificação necessária e o salário acima do mínimo nacional, cada unidade operacional da Fitolog trabalha com apenas um operador. O critério Aspectos Administrativos subdivide-se em dois subcritérios, Parcerias e Controle Administrativo. O subcritério Parcerias refere-se à utilização da estrutura dos próprios clientes onde são realizados os tratamentos, minimizando custos fixos (aluguel, água, energia) com o estabelecimento de postos avançados. O subcritério Controle Administrativo leva em conta o subcritério anterior acrescido da distância do local do tratamento até a sede administrativa da Fitolog (Porto Alegre). Quanto mais próxima da capital gaúcha, e melhor o relacionamento com o parceiro, teoricamente, melhor será o controle administrativo sobre as operações. Este subcritério tem fundamental importância na proposta de operações com unidades descentralizadas. O critério Custo dos Insumos subdivide-se em dois subcritérios, GLP (fonte de calor dos módulos de aquecimento dos maquinários da empresa) e Óleo Diesel (para transporte da unidade móvel), os dois itens mais importantes na prestação do serviço pelas unidades operacionais móveis. O critério Geografia diz respeito, fundamentalmente, à distância do local de instalação da unidade móvel em relação ao centro de manutenção dos equipamentos e veículos da empresa (subcritério Proximidade da Assistência Técnica) e as distâncias médias percorridas pelas unidades móveis para atendimentos de toda a demanda de tratamentos mensais dos clientes (subcritério Proximidade de Mercados Consumidores). Este subcritério é muito importante, porque o deslocamento até o cliente é um dos maiores custos da empresa na realização de tratamentos fitossanitários. As distâncias foram calculadas utilizando o Google Maps (2010). O último nível, composto pelas cidades potencialmente escolhidas por consenso entre os executivos da empresa, representa as alternativas de decisão.

\subsection{Matrizes de comparação}

Na sequência, de acordo com a técnica AHP e a estrutura hierárquica da Figura 3, todos os pares de comparações entre os objetos de cada nível hierárquico com respeito ao objeto no nível hierárquico imediatamente acima foram produzidos pelos executivos e incorporados em matrizes de escores. Em reuniões sucessivas, buscaram-se consenso e validade aos valores atribuídos. 0 Microsoft ${ }^{\circ}$ Excel foi utilizado para facilitar a comunicação entre os participantes e registrar devidamente os resultados. Os resultados das comparações das alternativas com relação ao critério mão de obra e aos seis subcritérios são apresentados nos quadros 1 a 7 .

Quadro 1 - Comparação das alternativas com relação ao subcritério Mão de Obra

\begin{tabular}{|c|c|c|c|c|c|c|}
\hline & Caxias do Sul & Montenegro & Portão & Porto Alegre & Vale Real & $\begin{array}{c}\text { Prioridade } \\
\text { Relativa }\end{array}$ \\
\hline Caxias do Sul & 1 & 1 & $1 / 3$ & $1 / 4$ & $1 / 3$ & 0,080 \\
\hline Montenegro & 1 & 1 & $1 / 3$ & $1 / 3$ & $1 / 2$ & 0,086 \\
\hline Portão & 3 & 3 & 1 & $1 / 4$ & 1 & 0,225 \\
\hline
\end{tabular}




\begin{tabular}{|c|c|c|c|c|c|c|}
\hline & Caxias do Sul & Montenegro & Portão & Porto Alegre & Vale Real & $\begin{array}{c}\text { Prioridade } \\
\text { Relativa }\end{array}$ \\
\hline Porto Alegre & 4 & 3 & 4 & 1 & 3 & 0,409 \\
\hline \multirow[t]{2}{*}{ Vale Real } & 3 & 2 & 1 & $1 / 3$ & 1 & 0,200 \\
\hline & & & & & & C.R. $=0,044$ \\
\hline
\end{tabular}

Fonte: elaborado pelos autores.

Quadro 2 - Comparação das alternativas com relação ao subcritério Parcerias

\begin{tabular}{|c|c|c|c|c|c|c|}
\hline & Caxias do Sul & Montenegro & Portão & Porto Alegre & Vale Real & $\begin{array}{c}\text { Prioridade } \\
\text { Relativa }\end{array}$ \\
\hline Caxias do Sul & 1 & 1 & 2 & $1 / 6$ & $1 / 3$ & 0,100 \\
\hline Montenegro & 1 & 1 & 2 & $1 / 6$ & $1 / 2$ & 0,104 \\
\hline Portão & $1 / 2$ & $1 / 2$ & 1 & $1 / 8$ & $1 / 4$ & 0,053 \\
\hline Porto Alegre & 6 & 6 & 8 & 1 & 2 & 0,511 \\
\hline \multirow[t]{2}{*}{ Vale Real } & 3 & 2 & 4 & $1 / 2$ & 1 & 0,233 \\
\hline & & & & & & C.R. $=0,007$ \\
\hline
\end{tabular}

Fonte: elaborado pelos autores.

Quadro 3 - Comparação das alternativas com relação ao subcritério Controle Administrativo

\begin{tabular}{|c|c|c|c|c|c|c|}
\hline & $\begin{array}{l}\text { Caxias do } \\
\text { Sul }\end{array}$ & Montenegro & Portão & Porto Alegre & Vale Real & $\begin{array}{c}\text { Prioridade } \\
\text { Relativa }\end{array}$ \\
\hline Caxias do Sul & 1 & 1 & $1 / 2$ & $1 / 5$ & $1 / 3$ & 0,079 \\
\hline Montenegro & 1 & 1 & $1 / 2$ & $1 / 5$ & $1 / 3$ & 0,079 \\
\hline Portão & 2 & 2 & 1 & $1 / 4$ & $1 / 2$ & 0,150 \\
\hline Porto Alegre & 5 & 5 & 4 & 1 & 2 & 0,444 \\
\hline \multirow[t]{2}{*}{ Vale Real } & 3 & 3 & 2 & $1 / 2$ & 1 & 0,248 \\
\hline & & & & & & C.R. $=0,006$ \\
\hline
\end{tabular}

Fonte: elaborado pelos autores.

Quadro 4 - Comparação das alternativas com relação ao subcritério GLP

\begin{tabular}{|c|c|c|c|c|c|c|}
\hline & $\begin{array}{c}\text { Caxias do } \\
\text { Sul }\end{array}$ & Montenegro & Portão & Porto Alegre & Vale Real & $\begin{array}{c}\text { Prioridade } \\
\text { Relativa }\end{array}$ \\
\hline Caxias do Sul & 1 & 1 & 1 & $1 / 2$ & 1 & 0,167 \\
\hline Montenegro & 1 & 1 & 1 & $1 / 2$ & 1 & 0,167 \\
\hline Portão & 1 & 1 & 1 & $1 / 2$ & 1 & 0,167 \\
\hline Porto Alegre & 2 & 2 & 2 & 1 & 2 & 0,333 \\
\hline \multirow[t]{2}{*}{ Vale Real } & 1 & 1 & 1 & $1 / 2$ & 1 & 0,167 \\
\hline & & & & & & C.R. $=0,000$ \\
\hline
\end{tabular}

Fonte: elaborado pelos autores.

Quadro 5 - Comparação das alternativas com relação ao subcritério Diesel

\begin{tabular}{|c|c|c|c|c|c|c|}
\hline & $\begin{array}{c}\text { Caxias do } \\
\text { Sul }\end{array}$ & Montenegro & Portão & Porto Alegre & Vale Real & $\begin{array}{c}\text { Prioridade } \\
\text { Relativa }\end{array}$ \\
\hline Caxias do Sul & 1 & 1 & 1 & 1 & 1 & 0,200 \\
\hline Montenegro & 1 & 1 & 1 & 1 & 1 & 0,200 \\
\hline Portão & 1 & 1 & 1 & 1 & 1 & 0,200 \\
\hline Porto Alegre & 1 & 1 & 1 & 1 & 1 & 0,200 \\
\hline \multirow[t]{2}{*}{ Vale Real } & 1 & 1 & 1 & 1 & 1 & 0,200 \\
\hline & & & & & & C.R. $=0,000$ \\
\hline
\end{tabular}

Fonte: elaborado pelos autores. 
Quadro 6 - Comparação das alternativas com relação ao subcritério Proximidade da Assistência Técnica

\begin{tabular}{|c|c|c|c|c|c|c|}
\hline & $\begin{array}{l}\text { Caxias do } \\
\text { Sul }\end{array}$ & Montenegro & Portão & Porto Alegre & Vale Real & $\begin{array}{c}\text { Prioridade Re- } \\
\text { lativa }\end{array}$ \\
\hline Caxias do Sul & 1 & 4 & 3 & 1 & 5 & 0,349 \\
\hline Montenegro & $1 / 4$ & 1 & $1 / 2$ & $1 / 4$ & 2 & 0,100 \\
\hline Portão & $1 / 3$ & 2 & 1 & $1 / 3$ & $1 / 2$ & 0,104 \\
\hline Porto Alegre & 1 & 4 & 3 & 1 & 5 & 0,349 \\
\hline \multirow[t]{2}{*}{ Vale Real } & $1 / 5$ & $1 / 2$ & 2 & $1 / 5$ & 1 & 0,097 \\
\hline & & & & & & C.R. $=0,078$ \\
\hline
\end{tabular}

Fonte: elaborado pelos autores.

Quadro 7 - Comparação das alternativas com relação ao subcritério Proximidade de Mercados Consumidores

\begin{tabular}{|c|c|c|c|c|c|c|}
\hline & $\begin{array}{l}\text { Caxias do } \\
\text { Sul }\end{array}$ & Montenegro & Portão & Porto Alegre & Vale Real & $\begin{array}{c}\text { Prioridade Re- } \\
\text { lativa }\end{array}$ \\
\hline Caxias do Sul & 1 & $1 / 4$ & $1 / 8$ & $1 / 4$ & $1 / 6$ & 0,041 \\
\hline Montenegro & 4 & 1 & $1 / 2$ & 1 & 3 & 0,216 \\
\hline Portão & 8 & 2 & 1 & 2 & 3 & 0,364 \\
\hline Porto Alegre & 4 & 1 & $1 / 2$ & 1 & 2 & 0,193 \\
\hline \multirow[t]{2}{*}{ Vale Real } & 6 & $1 / 3$ & $1 / 3$ & $1 / 2$ & 1 & 0,186 \\
\hline & & & & & & C.R. $=0,045$ \\
\hline
\end{tabular}

Fonte: elaborado pelos autores.

No caso em estudo, os três critérios que possuem subdivisões subdividem-se cada um em apenas dois subcritérios. Portanto, a determinação das prioridades entre os subcritérios com relação a cada critério é obtida de modo direto, sem necessidade de elaboração de matrizes de comparação. $O$ Quadro 8 apresenta o sentimento do grupo de executivos encarregados da avaliação.

Quadro 8 - Prioridade relativa entre subcritérios em relação a cada critério

\begin{tabular}{|l|l|c|}
\hline \multirow{2}{*}{ Critério } & Subcritério & $\begin{array}{c}\text { Prioridade } \\
\text { relativa }\end{array}$ \\
\hline \multirow{2}{*}{ Aspectos administrativos } & Parcerias & 0,600 \\
\cline { 2 - 3 } Custo de insumos & Controle administrativo & 0,400 \\
\hline \multirow{2}{*}{ Geografia } & GLP & 0,400 \\
\cline { 2 - 3 } & Prosel & 0,600 \\
\cline { 2 - 3 } & Proximidade da assistência técnica & 0,100 \\
\hline
\end{tabular}

Fonte: elaborado pelos autores.

Finalmente, os resultados da comparação entre os critérios com relação ao objetivo da análise são apresentados no Quadro 9.

Quadro 9 - Comparação dos critérios em relação ao objetivo

\begin{tabular}{|c|c|c|c|c|c|}
\hline & Mão de Obra & $\begin{array}{c}\text { Aspectos Ad- } \\
\text { ministrativos }\end{array}$ & $\begin{array}{c}\text { Custo dos In- } \\
\text { sumos }\end{array}$ & Geografia & $\begin{array}{c}\text { Prioridade } \\
\text { relativa }\end{array}$ \\
\hline Mão de Obra & 1 & $1 / 3$ & 1 & $1 / 9$ & 0,071 \\
\hline $\begin{array}{c}\text { Aspectos Administra- } \\
\text { tivos }\end{array}$ & 3 & 1 & 3 & $1 / 3$ & 0,214 \\
\hline Custo dos Insumos & 1 & $1 / 3$ & 1 & $1 / 9$ & 0,071 \\
\hline Geografia & 9 & 3 & 9 & 1 & 0,643 \\
\hline & \multicolumn{7}{|r|}{} & & & C.R. $=\mathbf{0 , 0 0 0}$ \\
\hline
\end{tabular}

Fonte: elaborado pelos autores. 


\subsection{Verificação de consistência}

Na seção anterior, foram apresentados os resultados finais consensuais do grupo de trabalho. Várias reuniões foram realizadas visando avaliar a consistência interna das comparações realizadas, incluindo os casos das matrizes de comparação das alternativas que apresentaram inconsistências (CR superior a 0,1). Confrontado com tais inconsistências, o grupo aprofundou a análise, revisando suas avaliações e obtendo finalmente um modelo consistente.

\subsection{Determinação dos escores cardinais finais das alternativas}

Após os passos descritos nas seções anteriores, os pesos relativos distribuíram-se como apresentado no Quadro 10.

Quadro 10 - Prioridades compostas das alternativas

\begin{tabular}{|c|c|c|c|c|c|}
\hline & $\begin{array}{c}\text { Mão de obra } \\
(\mathbf{0 , 0 7 1 )}\end{array}$ & $\begin{array}{c}\text { Aspectos adminis- } \\
\text { trativos } \\
\mathbf{( 0 , 2 1 4 )}\end{array}$ & $\begin{array}{c}\text { Custo dos in- } \\
\text { sumos } \\
(\mathbf{0 , 0 7 1 )}\end{array}$ & $\begin{array}{c}\text { Geografia } \\
\mathbf{( 0 , 6 4 3 )}\end{array}$ & Composição \\
\hline Caxias do Sul & 0,079 & 0,088 & 0,187 & 0,074 & $\mathbf{0 , 0 8 5}$ \\
\hline Montenegro & 0,092 & 0,092 & 0,187 & 0,218 & $\mathbf{0 , 1 8 0}$ \\
\hline Portão & 0,193 & 0,088 & 0,187 & 0,358 & $\mathbf{0 , 2 7 6}$ \\
\hline Porto Alegre & 0,455 & 0,492 & 0,253 & 0,220 & $\mathbf{0 , 2 9 7}$ \\
\hline Vale Real & 0,182 & 0,240 & 0,187 & 0,130 & $\mathbf{0 , 1 6 2}$ \\
\hline C.R. & $\mathbf{0 , 0 4 4}$ & $\mathbf{0 , 0 0 7}$ & $\mathbf{0 , 0 0 0}$ & $\mathbf{0 , 0 4 9}$ & $\mathbf{0 , 0 3 6}$ \\
\hline
\end{tabular}

Fonte: elaborado pelos autores.

O resultado da priorização das alternativas, obtido através da aplicação da técnica AHP, reflete a escolha do grupo de trabalho. Como primeira opção de localização está a cidade de Porto Alegre, seguida de perto pela cidade de Portão e, na sequência, Montenegro, Vale Real e Caxias do Sul.

A comparação entre os pesos relativos, dados aos critérios gerais, demonstra que o fator preponderante foi a Geografia $(0,643$ contra 0,$071 ; 0,214$ e 0,071$)$, composta pela Proximidade de Mercados Consumidores e Assistência Técnica. Nota-se que Portão é uma excelente localidade no aspecto geográfico, inclusive melhor que Porto Alegre. Entretanto, não se destaca nos demais aspectos, fazendo emergir Porto Alegre como a melhor opção.

\section{CONSIDERAÇÕES FINAIS}

Definido por Moreira (2008) como um problema comum, embora complexo e crucial para várias organizações, a localização de um novo empreendimento (ou a relocalização de um empreendimento já existente) é facilitada se o tomador de decisão tem um modelo bem estruturado, a fim de comparar as opções de localização pré-selecionadas. Ao permitir o agrupamento em uma mesma estrutura lógica de aspectos qualitativos e quantitativos, a técnica AHP pareceu a melhor escolha para resolver o problema de localização da Fitolog.

Antes de usar a técnica AHP, como auxiliar do processo decisório, a avaliação das alternativas encaminhava-se para uma análise baseada apenas em aspectos quantitativos e financeiros, como custo dos insumos e distâncias a percorrer (clientes mais afastados significam custos de transporte mais elevados para as unidades operacionais móveis). Por esta razão, a decisão da empresa convergia para a escolha de uma cidade mais centralizada em relação ao grupo de clientes, como: Portão, Vale Real ou Montenegro (ver Figura 4). 


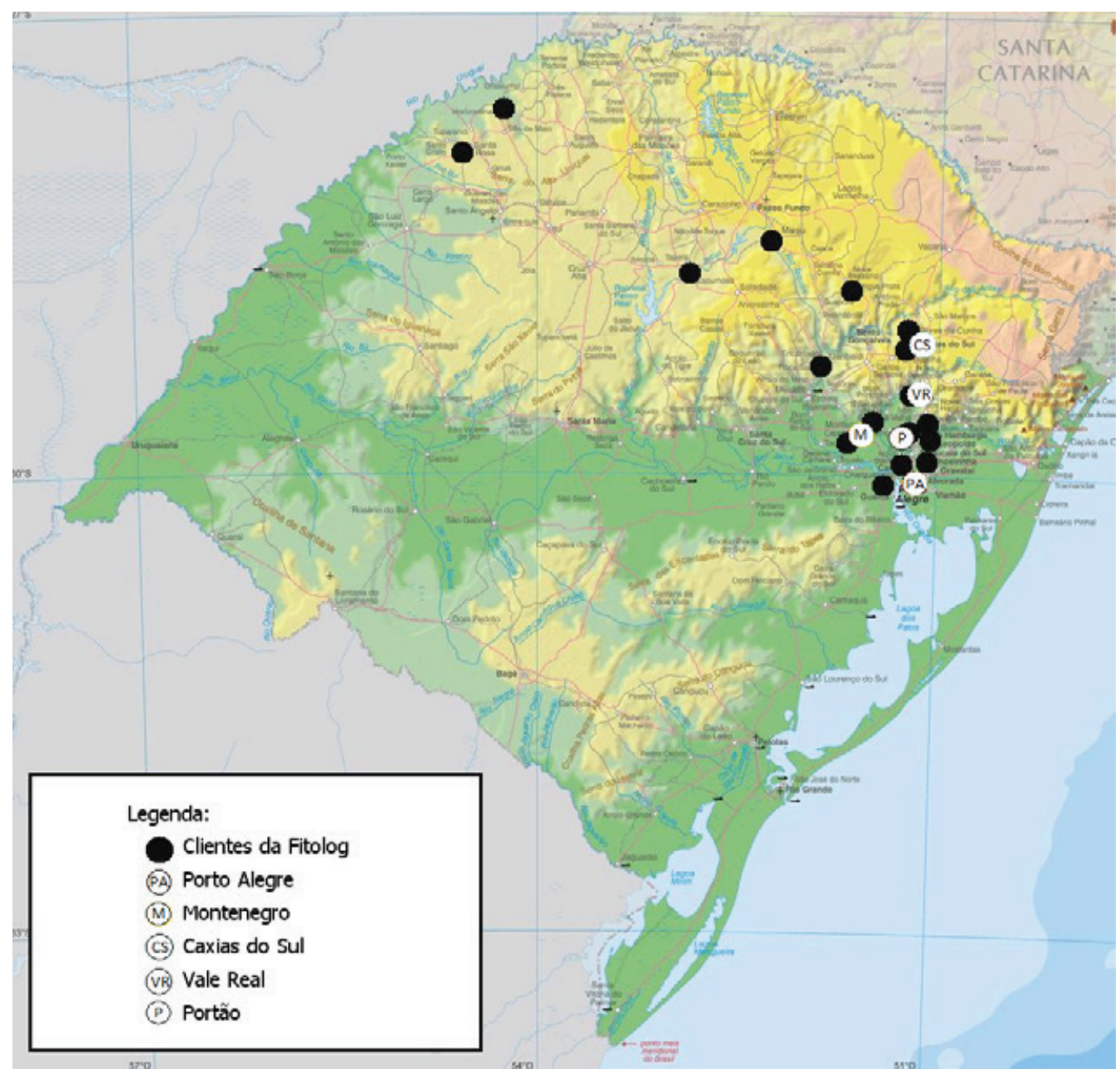

Figura 4 - Localização dos principais clientes da Fitolog e das cidades pré-selecionadas (IBGE, 2011).

A análise baseada na técnica AHP, entretanto, tornou claro que esta linha de pensamento era equivocada, pois a técnica apontou a cidade de Porto Alegre como a mais interessante na composição geral, para basear as unidades operacionais móveis da empresa. Isto se dá porque a cidade apresenta uma série de benefícios qualitativos, que estavam sendo deixados de fora na análise estritamente financeira, como disponibilidade e necessidade de treinamento de mão de obra e melhor controle administrativo. Outro aspecto julgado interessante pelos executivos envolvidos no processo, ao usar a técnica AHP, foi sua habilidade de quantificar a avaliação dos aspectos qualitativos e o consequente agrupamento de todos os critérios em um número final, criando uma ordenação global de preferência das cidades e várias outras ordenações paralelas de critérios e subcritérios.

Como a Fitolog atualmente possui três unidades operacionais móveis, ela pode decidir localizá-las todas na cidade com o escore global mais elevado (Porto Alegre), ou descentralizá-las, localizando cada uma delas em uma cidade distinta, com características complementares. Por exemplo, poderia ser interessante localizar uma unidade na cidade mais centralizada (Portão) em relação ao grupo de consumidores, enquanto as duas outras unidades poderiam ser localizadas onde a mão de obra e os aspectos administrativos têm melhor qualificação (Porto Alegre). Conclui-se, pois, que a aplicação da técnica AHP como modelo de avaliação das localizações alternativas ofereceu aos executivos da Fitolog excelentes informações, fundadas em dados consistentes e baseadas em uma análise multicritério, que pode guiá-los com segurança nas decisões com relação com relação 
à definição da melhor combinação de localizações de suas unidades operacionais móveis.

Entretanto, deve-se observar que, embora a análise aponte caminhos importantes para a otimização logística através da realocação das unidades operacionais móveis, não pode ter seus resultados tomados como "a situação ótima final", haja vista que as possibilidades para estabelecimento de postos avançados dentro do Estado do Rio Grande do Sul são praticamente ilimitadas, considerando o total de municípios existentes. Uma ótima oportunidade para um trabalho futuro seria explorar, sob a ótica de logística de operações, combinações de deslocamento entre municípios, visando minimizar tempo e custos de combustível. Apesar disso, principalmente no caso de pequenas empresas, soluções criativas, como a apresentada neste estudo, que prevê a possibilidade de localização de unidades operacionais móveis da Fitolog na estrutura física de parceiros, eliminando custos fixos do estabelecimento de novas filiais, são fundamentais para que jovens empresas atinjam um crescimento contínuo e sustentável.

Os autores agradecem ao CNPq e à CAPES pelo apoio à pesquisa na qual este trabalho se baseia.

\section{REFERENCIAS}

ABREU, L. M.; GRANEMANN, S. R.; GARTNER, I.; BERNARDES, R. S. Escolha de um programa de controle da qualidade da água para consumo humano: aplicação do método AHP. Revista Brasileira de Engenharia Agrícola e Ambiental, v. 4 , n. 2, p. 257-262, 2000

ACKOFF, R. L.; SASIENI, M. Fundamentals of operations research. New York: John Wiley \& Sons, 1968.

AGUIAR, D. C.; SALOMON, V. A. P. Avaliação da prevenção de falhas em processos utilizando métodos de tomada de decisão. Produção, v. 17, n. 3, p. 502-519, 2007.

ATTHIRAWONG, W.; MACCARTHY, B. An application of the Analytical Hierarchy Process to international location decision-making. Synthesis, v.8, n.3/4, p. 1-18, 2002.

BADRI, M. A. Combining the analytic hierarchy process and goal programming for global facility location-allocation problem. International Journal of Production Economics, v. 62, p. 237-248, 1999.

BANDEIRA, D. L.; BECKER, J. L.; ROCHA, A. K. Sistemática multicritério para priorização de embarques marítimos. Revista de Administração Mackenzie, v. 11, n. 6, p. 107-130, 2010.
BARIN, A.; CANHA, L. N.; ABAIDE, A. R.; MAGNAGO, K. F.; WOTTRICH, B.; MACHADO, R. Q. Multiple criteria analysis for energy storage selection. Energy and Power Engineering, v.3, p. 557-564, 2011.

BARROS, M. A.; MOREIRA, M. A.; RUDORFF, B. F. T. Processo analítico hierárquico na identificação de áreas favoráveis ao agroecossistema cafeeiro em escala municipal. Revista Agropecuária Brasileira, v. 42, n. 12, p. 1769-1777, 2007.

BERNARDON, D. P.; SPERANDIO, M.; GARCIA, V. J.; CANHA, L. N.; ABAIDE, A. R.; DAZA, E. F. $B$. AHP decision-making algorithm to allocate remotely controlled switches in distribution networks. IEEE Transactions on Power Delivery, v. 26, n. 3, p. 1884-1892, 2011.

BRYAN, D. L.; O'KELLY, M. E. Hub-and-spoke networks in air transportation: an analytical review. Journal of Regional Science, v. 39, n. 2, p. 275-295, 1999.

CAMPOS, V. B. G.; RAMOS, R. A. R.; CORREIA, D. M. S. Multi-criteria analysis procedure for sustainable mobility evaluation in urban areas. Journal of Advanced Transportation, v. 43, n. 4, p. 371-390, 2008.

CARVALHO, C. M.; RIEDEL, P. S. Análise da suscetibilidade a escorregamentos nos entornos dos polidutos de Cubatão-SP, através 
de técnicas de informação geográfica. Holos Environment, v. 4, n. 2, p. 157-173, 2004.

CAVALCANTE, D. G.; PINHEIRO, E. S.; MACEDO, M. A.; MARTINOT, J. F.; NASCIMENTO, A. Z. A.; MARQUES, J. P. C. Análise da vulnerabilidade ambiental de um fragmento florestal urbano na Amazônia: Parque Estadual Sumaúma. Sociedade \& Natureza, v. 22, n. 2, p. 391-403, 2010.

CORSO, K. B.; LÖBLER, M. L. Understanding the subject's behavior in the interaction with a decision support system under time pressure and missing information. Journal of Information Systems and Technology Management, v. 8, n. 3, p. 581-604, 2011.

COSTA, H. G.; MOLL, R. N. Emprego do método de análise hierárquica (AHP) na seleção de variedades para o plantio de cana-de-açúcar. Gestão \& Produção, v. 6, n. 3, p. 243-256, 1999.

CRUZ JR., A. T.; CARVALHO, M. M. Obtenção da voz do consumidor: estudo de caso em um hotel ecológico. Produção, v. 13, n. 3, p. 88-100, 2003.

CUNHA, E. R. S. P..; OLIVEIRA, M. V.; SILVA, F. R. M. Utilização da técnica de processo analítico hierárquico (AHP) na avaliação da "favorabilidade" para a prospecção mineral de cromo na região de Pinheiros Altos, município de Piranga, MG, Brasil. Revista da Escola de Minas, v. 54, n. 2, p. 127-132, 2001.

DE CARLI, P. C.; DELAMARO, M. C.; SALOMON, V. A. P. Identificação e priorização dos fatores críticos de sucesso na implantação de fábrica digital. Produção, v. 20, n. 4, p. 549-564, 2010.

FARAHANI, R. Z.; ASGARI, N.; HEIDARI, N.; HOSSEININIA, M.; GOH, M. Covering problems in facility location: a review. Computers \& Industrial Engineering, v. 62, p. 368-407, 2012.

FITOLOG. Fitolog Controle de Pragas Ltda. Institucional, 2012a. Disponível em: <http://www.fitosystem.com.br/empresa/ institucional/?lang=pt>. Acesso em: 4 set. 2012.

FITOLOG. Fitolog Controle de Pragas
Ltda. Histórico, 2012b. Disponível em: <http://www.fitosystem.com.br/empresa/ historico/?lang=pt $>$. Acesso em: 4 set. 2012.

FOGLIATTO, F. S.; ALBIN, S. L. A hierarquical method for evaluating products with quantitative and sensory characteristics. IIE Transactions, v. 33, p. 1081-1092, 2001.

FLEURY, P. F.; WANKE, P.; FIGUEIREDO, K, F. Logística empresarial: a perspectiva brasileira. São Paulo: Atlas, 2000.

GARTNER, I. R.; MOREIRA, T. B. S.; GALVES, H. M. Análise do risco setorial como instrumento de controle gerencial em instituições financeiras. Revista de Administração Mackenzie, v. 10, n. 5, p. 107-129, 2009.

GOLDEN, B. L.; WASIL, E. A.; HARKER, P. T. The analytic hierarchy process: applications and studies. Berlim: Springer-Verlog, 1989.

GOMES, L. F. A. M.; ANDRADE, R. M. Performance evaluation in assets management with the AHP. Pesquisa Operacional, v. 32, n. 1, p. 31-53, 2012.

GOOGLE MAPS, 2010. Disponível em: <http:// maps.google.com.br/>. Acesso em: 2 nov. 2010.

HALE, T. S.; MOBERG, C. R. Location science research: a review. Annals of Operations Research, v. 123, p. 21-35, 2003.

HO, W. Integrated analytic hierarchy process and its applications - a literature review. European Journal of Operational Research, $\mathrm{v}$. 186, n. 1, p. 211-228, 2008.

IAÑEZ, M. M.; CUNHA, C. B. Uma metodologia para a seleção de um provedor de serviços logísticos. Produção, v. 16, n. 3, p. 394-412, 2006.

IBGE. Instituto Brasileiro de Geografia e Estatística, 2011. Disponível em: <http://www. ibge.gov.br/ibgeteen/mapas/>. Acesso em: 10 mar. 2011.

KORPELA, J.; TUOMINEN, M. A decision aid 
in warehouse site selection. International Journal of Production Economics, v. 45, p. 169-180, 1996.

KUO, R. J.; CHI, S. C.; KAO, S. S. A decision support system for locating convenience store through fuzzy AHP. Computers \& Industrial Engineering, v. 37, p. 323-326, 1999.

LAURINDO, F. J. B.; CARVALHO, M. M.; PESSÔA, M. S. P.; SHIMIZU, T. Selecionando uma aplicação de tecnologia da informação com enfoque na eficácia: um estudo de caso de um sistema para PCP. Gestão \& Produção, v. 9, n. 3, p. 377-396, 2002.

LIMA, J. P.; RAMOS, R. A. R.; FERNANDES JR., J. L. Priorização de pavimentos com uso da metodologia de análise multicritério e SIG. Engevista, v. 10, n. 2, p. 132-144, 2008.

LUZ, S. O. C.; SELLITTO, M. A.; GOMES, L. P. Medição de desempenho ambiental baseada em método multicriterial de apoio à decisão: estudo de caso na indústria automotiva. Gestão \& Produção, v. 13, n. 3, p. 557-570, 2006.

MACEDO-SOARES, T. D. L. v. A.; FIGUEIRA, L. A. P. A. Gestão estratégica da energia nucleoelétrica no Brasil: recursos e competências críticos para seu sucesso. Revista de Administração Contemporânea, v. 11, n. spe1, p. 55-76, 2007.

MARCHEZETTI, A. L.; KAVISKI, E.; BRAGA, M. C. B. Aplicação do método AHP para a hierarquização das alternativas de tratamento de resíduos sólidos domiciliares. Ambiente Construído, v. 11, n. 2, p. 173-187, 2011.

MATOS, D. M.; MOURA H. J. Proposta de um modelo para determinação do custo de capital baseado em análise hierárquica. Revista de Administração Contemporânea, v. 7, n. 4, p. 119-139, 2003.

MELO, M. T.; NICKEL, S.; SALDANHA-DAGAMA, F. Facility location and supply chain management: a review. European Journal of Operational Research, v. 196, p. 401-412, 2009.
MESA, J. A.; BOFFEY, T. B. A review of extensive facility location in networks. European Journal of Operational Research, v. 95, p. 592-603, 1996.

MOREIRA, D. A. Administração da produção e operações. São Paulo: Cengage Learning, 2008.

PADOVANI, M.; CARVALHO, M. M.; MUSCAT, A. R. N. Seleção e alocação de recursos em portfólio de projetos: estudo de caso no setor químico. Gestão \& Produção, v. 17, n. 1, p. 157-180, 2010.

PORTUGAL, L. S.; MORGADO, A. V.; LIMA JR., $O$. Location of cargo terminals in metropolitan areas of developing countries: the Brazilian case. Journal of Transport Geography, v. 19, n. 4, p. 900-910, 2011.

PREÇO DOS COMBUSTíVEIS. Preço dos combustíveis - Brasil, 2010. Disponível em <http://www.precodoscombustiveis.com.br/ mapa/busca>. Acesso em: 2 nov. 2010.

RAFAELI, L.; MÜLLER, C. J. Estruturação de um índice consolidado de desempenho utilizando o AHP. Gestão \& Produção, v. 14, n. 2, p. 363-377, 2007.

REIS, E.; LÖBLER, M. L. O processo decisório descrito pelo indivíduo e representado nos sistemas de apoio à decisão. Revista de Administração Contemporânea, v. 16, n. 3, p. 397-417, 2012.

REVELLE, C. S.; EISELT, H. A. Location analysis: a synthesis and survey. European Journal of Operational Research, v. 165, p. 1-19, 2005.

REVELLE, C. S.; EISELT, H. A.; DASKIN, M. S. A bibliography for some fundamental problem categories in discrete location science. European Journal of Operational Research, v. 184, p. 817-848, 2008.

RIBEIRO, D. D. M.; ROCHA, W. J. S. F.; GARCIA, A. J. V. Modelagem da potencialidade hídrica das águas subterrâneas da sub-bacia do rio Siriri, Sergipe, Brasil, com base em sistema de informações geográficas e técnicas de sensoriamento remoto. Ambiente \& Água, v. 
6, n. 2, p. 206-231, 2011.

RIBEIRO, L. S.; PASSOS, A. C.; TEIXEIRA, M. G. Seleção de tecnologias de comunicações no Exército Brasileiro utilizando os métodos multicritério de análise hierárquica, TODIM e software Sapiens. Produção, v. 22, n. 1, p. 132141, 2012.

ROSA, E. P. S.; SELLITTO, M. A.; MENDES, L. W. Avaliação multicriterial de desempenho e separação em aglomerados de fornecedores críticos de uma manufatura OKP. Produção, v. 16, n. 3, p. 413-428, 2006.

SAATY, T.L. The Analytic Hierarchy Process: planning, priority setting, resource allocation. New York: McGraw-Hill, 1980.

ŞAHIN, G.; SÜRAL, H. A review of hierarchical facility location models. Computers \& Operations Research, v. 34, p. 2310- 2331, 2007.

SAWICKA, H.; WĘGLIŃSKI, S.; WITORT, P. Application of multiple criteria decision aid methods in logistic systems. LogForum, v. $6, n$. 3, p. 99-110, 2010.

SELLITTO, M. A.; MENDES, L. W. Avaliação comparativa do desempenho de três cadeias de suprimentos em manufatura. Produção, v. 16, n. 3, p. 552-568, 2006.

SElLITTO, M. A.; WALTER, C. Avaliação do desempenho de uma manufatura de equipamentos eletrônicos segundo critérios de competição. Produção, v. 16, n. 1, p. 34-47, 2006.

SILVA, B.; FINARDI, C.; FORNECK, M.; SELLITTO, M. A. Análise comparativa e avaliação de prioridades de competição em três cadeias de suprimentos do setor petroquímico. Produção, v. 22, n. 2, p. 225-236, 2012.

SNYDER, L. V. Facility location under uncertainty: a review. IIE Transactions, v. 38, p. 537-554, 2006.
TÁlAMO, J. R.; CARVALHO, M. M. Seleção dos objetivos fundamentais de uma rede de cooperação empresarial. Gestão \& Produção, v. 11, n. 2, p. 239-250, 2004.

TORTORELLA, G. L.; FOGLIATTO, F. S. Planejamento sistemático de layout com apoio de análise de decisão multicritério. Produção, v. 18, n. 3, p. 609-624, 2008.

VAIDYA, O. S.; KUMAR, S. Analytic hierarchy process: an overview of applications. European Journal of Operational Research, v. 169, n. 1, p. 1-29, 2006.

VALENTE, R. O. A.; VETTORAZZI, C. A. Definition of priority areas for forest conservation through the ordered weighted averaging method. Forest Ecology and Management, v. 256, p. 1408-1417, 2008.

VENTURA, K. S.; REIS, L. F. R.; TAKAYANAGUI, A. M. M. Avaliação do gerenciamento de resíduos de serviços de saúde por meio de indicadores de desempenho. Engenharia Sanitária e Ambiental, v. 15, n. 2, p. 167-176, 2010.

WAGNER, Harvey M. Pesquisa operacional. Rio de Janeiro: Prentice-Hall do Brasil, 1986.

YANG, J.; LEE, H. An AHP decision model for facility location selection. Facilities, v. 15, n. 9, p. 241-254, 1997.

YURIMOTO, S.; MASUI, T. Design of a decision support system for overseas plant location in the EC. International Journal of Production Economics, v. 41, p. 411-418, 1995. 